\title{
MANEJO ESTOMATOLÓGICO DE UN PACIENTE PEDIÁTRICO CON DISTROFIA MUSCULAR DE DUCHENNE. REPORTE DE UN CASO
}

\section{STOMATOLOGIC MANAGEMENT OF A CHILD PATIENT WITH DUCHENNE MUSCULAR DYSTROPHY. CASE REPORT}

\author{
Od. Esp. Alvear María. ${ }^{1 *}$,Od. Esp. Mendoza Roberto ${ }^{2}$, Od.Esp. Alaniz Aydee ${ }^{3}$ \\ ${ }^{1}$ Especialista en Odontopediatría. Catedrático Universidad Católica de Cuenca.Ecuador. \\ ${ }^{2}$ Especialista en Odontopediatría. Catedrático de la Especialidad de Odontopediatría Universidad Nacional Autónoma de \\ México.México. \\ ${ }^{3}$ Especialista en Odontopediatría. Catedrático en la Universidad Justo Sierra. México DF.México. \\ *cristhialvear@hotmail.com
}

\begin{abstract}
Resumen
OBJETIVO: La Distrofia muscular de Duchenne (DMD) es una enfermedad ligada al cromosoma X, que afecta aproximadamente 1 de cada 3,300 varones nacidos vivos. Es causada por la ausencia o la mutación del gen que codifica la proteína distrofina. El propósito de este caso fue revisar la literatura actual sobre los aspectos importantes de atención infantil en la DMD. PRESENTACIÓN DE CASO: Acude a la clínica de Odontopediatría, un paciente masculino de 8 años de edad con discapacidad para la marcha y constitución ectomorfica, diagnosticado médicamente de DMD. A la exploración intraoral se encontró: clase I de Angle, mordida borde a borde anterior, diastemas, apiñamiento anteroinferior, gingivitis localizada, caries activas y macroglosia relativa. El manejo estomatológico fue realizado en cuatro fases: Preventiva, Restauradora, Quirúrgica y Mantenimiento. Se logró gran éxito clínico, requiriendo de un trabajo multidisciplinario, para proporcionar resultados satisfactorios a largo plazo que incrementen la calidad de vida del paciente.
\end{abstract}

Palabras clave: Distrofia muscular de duchenne, odontopediatría, distrofina.

\begin{abstract}
AIM: Duchenne muscular dystrophy (DMD) is an X linked condition that affects approximately 1 in 3300 live male births. It is caused by the absence or disruption of the protein dystrophin. The purpose of this case was to review the current literature about the important aspects of the DMD in children. CASE PRESENTATION: An 8 year old male patient arrives to the pediatric dentistry clinic with a walking disability and ectomorphic constitution, diagnosed with DMD. At the intraoral examination was found: neutrocclusion, edge-to-edge incisor relationship, diastemas, lower anterior crowding, localized gingivitis, active caries lesions and relative macroglossia. The stomatologic management was conducted in four phases: Preventive phase, Restorative phase, Surgical phase and Maintenance phase. A successful clinical approach was achieved, needing a highly specialized multidisciplinary work to provide satisfactory results that increases the quality of life of this patient.
\end{abstract}

Key words: Duchenne muscular dystrophy, pediatric dentistry, dystrophin.

\section{INTRODUCCIÓN}

La Distrofia Muscular de Duchenne (DMD) también conocida como Distrofia Muscular Progresiva, constituye un trastorno neuromuscular de carácter recesivo ligado al cromosoma $X$, resulta de una variedad de mutaciones en el gen que codifica la distrofina. ${ }^{1}$ La distrofina constituye una proteína subsarcolémica de gran tamaño, $427 \mathrm{kDa}$, cuya función es actuar como anclaje entre las proteínas citoesqueléticas y las proteínas integrales de membrana, previniendo el daño de la célula muscular durante el proceso de contracción. ${ }^{2}$ En el caso de los afectados por la DMD, los valores de distrofina se encuentran entre el 0-5\% de los rangos normales, la acusada disminución de la misma ocurre tras deleciones o duplicaciones a nivel del gen humano DMD ubicado en el brazo corto del cromosoma X, en el locus 21.2. ${ }^{1}$

Esta enfermedad afecta principalmente a individuos 
masculinos en una relación de 1 por cada 3, 000 a 6, 000 nacidos vivos, ${ }^{3}$ sin embargo en algunas situaciones puede afectar a mujeres a pesar de la naturaleza recesiva de la enfermedad, quienes son portadoras parcialmente afectadas, pero no con la misma severidad que los varones. ${ }^{1,4}$

Los síntomas por lo general aparecen antes de los 6 años de edad, pero también pueden presentarse en la infancia temprana. ${ }^{5}$ Se observa debilidad muscular progresiva de la pelvis y las piernas, la cual se asocia a pérdida de masa muscular. Inicialmente los músculos de la pantorrilla se agrandan y son finalmente reemplazados por grasa y tejido conectivo, razón por lo cual la enfermedad se conoce también como Distrofia Pseudohipertrófica de Duchenne. ${ }^{6}$ Se presentan contracturas musculares produciendo incapacidad para utilizar los músculos debido al acortamiento de las fibras y a la fibrosis del tejido conjuntivo. Hacia la edad de los 10 años, se requieren de prótesis ortopédicas para poder caminar y a la edad de 12 años, la mayoría de los pacientes están confinados a una silla de ruedas. ${ }^{3}$

Los huesos se desarrollan en forma anormal dando lugar a deformidades características entre las cuales destaca la escoliosis. $^{7}$ El $30 \%$ de los pacientes con DMD presentan discapacidad mental leve. En los estados avanzados de la enfermedad se presenta cardiomiopatía y trastornos respiratorios. ${ }^{1}$ Alrededor de la cavidad oral la distrofia muscular puede ser observada a menudo enmascarada por el crecimiento excesivo de los tejidos conectivo y adiposo. ${ }^{8,9}$ El proceso se evidencia con la hipertrofia del orbicular de los labios, masetero y músculos de la lengua.9 Defectos maxilares, maloclusión: mordida abierta y mordida cruzada son frecuentemente observadas a causa de los cambios en la forma de la base del cráneo. ${ }^{8}$ Adicionalmente encontramos un aplanamiento del paladar, ensanchamiento transversal de los arcos dentales, hipotonía lingual, disfagia y reflujo gastroesofágico. Los pacientes tienden a desarrollar un hábito parafuncional de respiración oral. ${ }^{10}$

La sospecha del diagnóstico de DMD debe ser considerada independientemente de los antecedentes familiares y suele estar desencadenada por los siguientes signos:

Problemas con la función muscular, signo de Gowers y retraso en el lenguaje verbal. Niveles séricos incrementados de la proteína muscular creatin fosfoquinasa (CPK) y de enzimas hepáticas AST y ALT (aspartato aminotransferasa y alanina aminotransferasa). ${ }^{11-13,16}$

El odontopediatra debe realizar un abordaje odontológico precoz e inmediato, priorizando aspectos preventivos para reducir la incidencia de caries en estos pacientes. Es necesario formular un programa preventivo fácil de seguir para el paciente y para quienes son responsables de él. ${ }^{14} \mathrm{Se}$ enfatiza que estos pacientes requieren de tratamiento ambulatorio estricto en varias citas, con el uso de anestésicos locales bajo interconsulta con el cardiólogo y con profilaxis antibiótica previa en los tratamientos que produzcan sangrado. El manejo conductual puede ser complicado por problemas

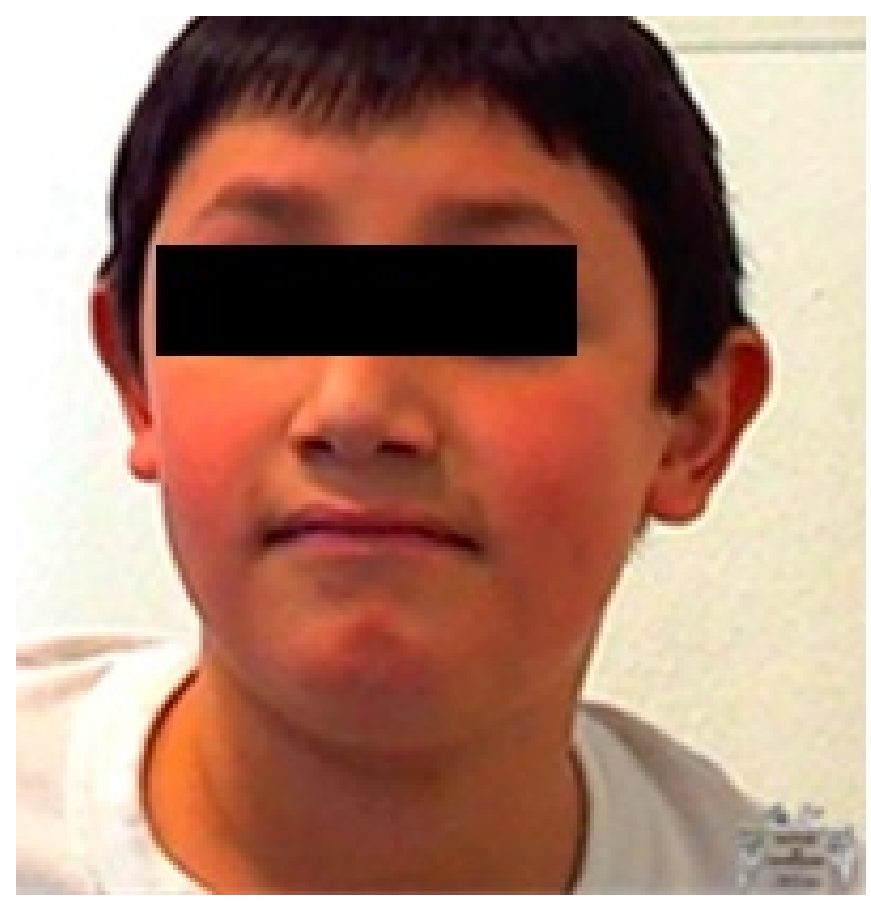

Fig. 1. A) Dolicofacial, ojos simétricos, desvío del mentón y cuello cilíndrico.

de comportamiento e infecciones respiratorias permanentes. El uso de anestesia general está contraindicado en la DMD por la atrofia de los músculos respiratorios, lo cual reduce la capacidad vital de los pulmones, predisponiendo a fallo respiratorio y complicaciones potencialmente graves en el periodo operatorio. ${ }^{9,17}$

\section{PRESENTACIÓN DEL CASO}

Se presenta a la clínica de odontopediatría, DEPeI, UNAM, un paciente masculino de 8 años de edad, con diagnóstico médico de Distrofia Muscular de Duchenne. Refiere dolor en la región dorsal, cefalea de predominio diurno, terrores nocturnos y apnea obstructiva del sueño. Niega sintomatología respiratoria o deglutoria, sin embargo describe presentar episodios arrítmicos repetidos, controlados. A la exploración física extraoral se observa un niño de edad aparentemente menor a la referida, integro, de constitución ectomorfica, poco cooperador de acuerdo a la clasificación de Frankl, incapaz de realizar marcha independiente por si mismo, utilizando una silla de ruedas para desplazarse. Presenta cráneo normocéfalo, aspecto dolicofacial, ojos simétricos, hipotonicidad del labio inferior, desvío del mentón hacia el lado izquierdo, implantación asimétrica de las orejas, cuello cilíndrico, ausencia de adenomegalias y fuerza muscular 4/4 global. (Fig.1)

Las extremidades superiores se encuentran integras con hipotrofia generalizada de predominio proximal, tono muscular disminuido en codos y muñecas. A la exploración 
A.

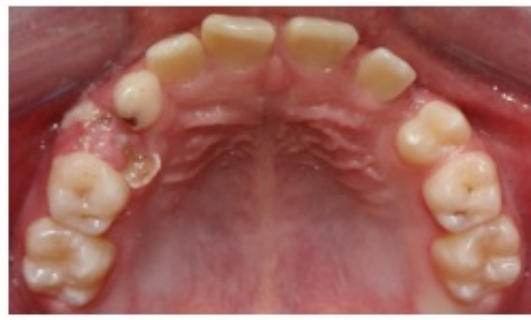

B.

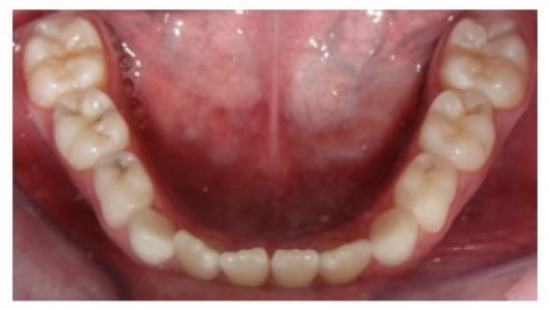

C.

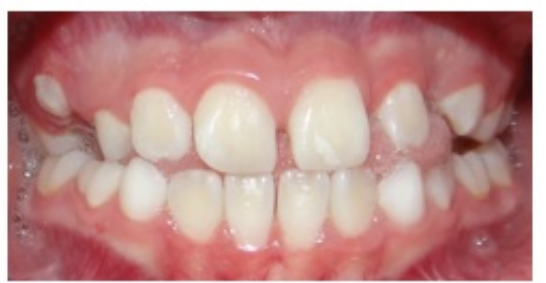

Fig. 2. A) Arco dentario superior elíptico, pérdida prematura del organó dentario 63 , resto radicular del 54, gingivitis inducida por placa bacteriana localizada y procesos de caries activos e inactivos. B) Arco dentario inferior elíptico con apiñamiento discreto en el sector anterior, procesos cariosos activos e inactivos. C) En las arcadas en oclusión se observa mordida borde a borde, diastema medio superior y ausencia clínica del organó dentario 63 (nótese la macroglosia relativa presente en el paciente).

intraoral se observa: arcada dentaria superior elíptica, dentición mixta segunda fase con perdida prematura de la pieza 6.3; resto radicular del 54 , gingivitis inducida por placa bacteriana localizada, procesos de caries activos e inactivos. Arcada dentaria inferior elíptica, dentición mixta primera fase con apiñamiento discreto en el sector anterior, procesos cariosos activos e inactivos. En las arcadas en oclusión se observa relación de neutroclusión molar bilateral, mordida borde a borde a borde, diastema medio superior, pérdida prematura del organó dentario 63 y macroglosia relativa . (Fig. 2). Se procedió a realizar el manejo odontológico en cuatro fases específicas:

\subsection{Fase Preventiva}

Al encontrar una función muscular deteriorada es evidente pensar que la capacidad de destreza del paciente es muy limitada, por lo que todas las instrucciones de higiene fueron dirigidas a sus padres. El paciente fue clasificado de alto riesgo de acuerdo a las normas internacionales del Cariesrisk Assessment Tool (CAT) de la Academia Americana de

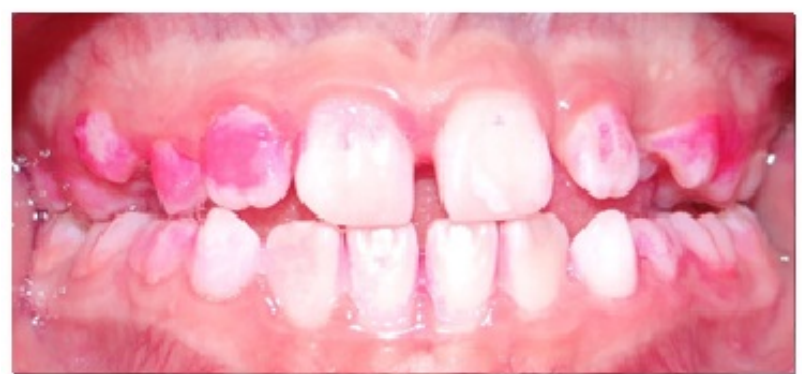

Fig. 3. Control mecánico de biopelículas orales, utilizando el Índice de Placa de O Leary para cuyo registro se emplea solución reveladora a base de eritrosina.

Odontopediatría , se procedió a establecer un programa preventivo específico e individualizado basado en las siguientes medidas terapéuticas:

1) Realizar la higiene oral diaria tres veces al día con un cepillo dental infantil de cerdas suaves, adaptado con un mango grueso que pueda ser sostenido por el niño.

2) Utilizar pasta dental fluorada que contenga $0,5 \%$ de fluoruro.

3) Dado el estado sistémico del paciente la capacidad de expectoración puede verse comprometida, por ello se recomienda utilizar diariamente un enjuague de fluoruro sódico al $0.05 \%$, el cual debe ser aplicado por la madre con un cotonete humedecido en el producto para evitar la ingesta del mismo. El uso de este agente se mantendrá durante 3 semanas del mes.

4) Utilizar digluconato de clorhexidina al $0.12 \%$ (spray o gel bioadhesivo) durante 1 semana del mes.

5) Implementar el uso de seda dental, después del cepillado tras previa capacitación con el odontopediatra.

6) Implementar el uso de polialcoholes como el xilitol. En la clínica de odontopediatría se realizó:

1) Control de placa bacteriana: Se repitieron los controles cada 3 semanas. (Fig.3).

2) Profilaxis dental con instrumentos rotatorios de baja velocidad. (Fig.4).

3) Aplicación profesional de fluoruro de sodio con trifosfato cálcico en barniz a una concentración del $5 \%$ (Clinpro White Varnish) cada tres meses. (Fig.5).

\subsection{Fase Restauradora:}

Fundamentada en la rehabilitación oportuna de lesiones cariosas, con el uso de técnicas mínimamente invasivas (Fig.6) .Los procedimientos restauradores realizados fueron los siguientes:

1) Selladores de fosetas y fisuras en 16, 26, 36, 46 y 84 : una vez aislados con dique de hule, se aplicó hipoclorito 


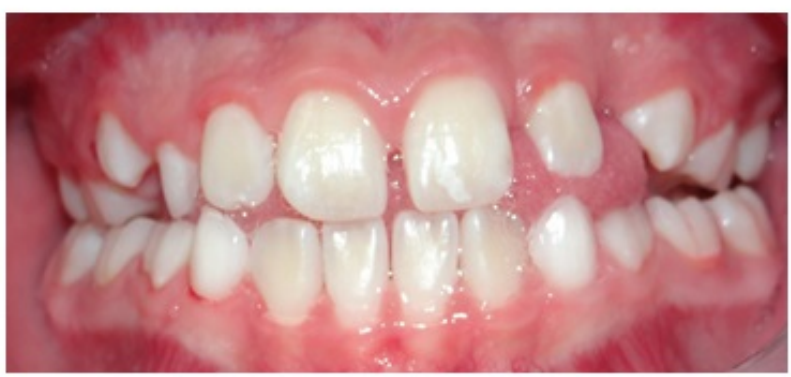

Fig. 4. Imagen clínica post profilaxis dental, para la cual se utilizó cepillo profiláctico, pasta profiláctica Clinpro e instrumentos rotatorios de baja velocidad.

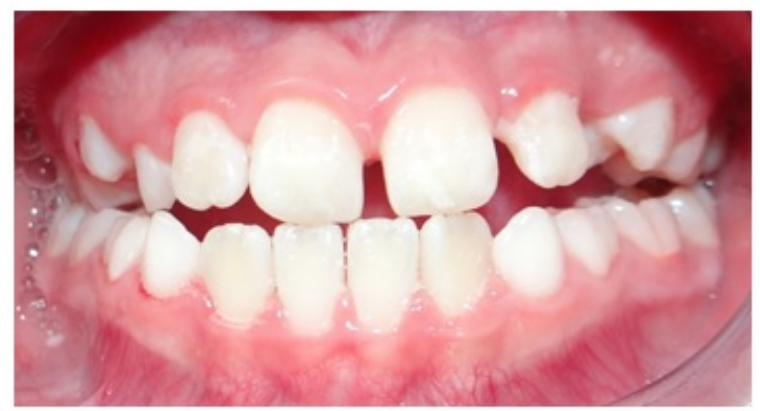

Fig. 5. Aplicación profesional de barniz de flúor al 5\% Clinpro White Varnish con trifosfato cálcico , nótese que el barniz no se elimina al fraguar y fluye efectivamente en las superficies de aplicación.

de sodio $(\mathrm{NaOCl})$ al $5.2 \%$ por 60 segundos (desproteinización), se grabó con ácido fosfórico (H3PO4) al $37 \%$ durante 15 segundos y se colocó el sellador en las superficies oclusales y palatinas.

2) Ionómero de vidrio modificado con resina en 55, 53, 65, 74,75 y 85: tras aislamiento previo con dique de hule y remoción de caries con pieza de alta velocidad, se secaron ligeramente las cavidades, se colocó el imprimador y se polimerizó por 10 segundos. Se procedió a adaptar el material restaurador Ketac N100,3M, dando forma y contorno a las restauraciones, luego de ello se polimerizó por 20 segundos. El pulido fue inmediato con instrumentos convencionales de acabado y bajo condiciones de humedad.

\subsection{Fase Quirúrgica}

Contempló la exploración radiográfica (Fig.7) y posterior extracción del organó dentario 54. Debido que el paciente presentaba historial de taquiarritmias, se realizó interconsulta con el cardiólogo para establecer la terapia farmacológica
A.

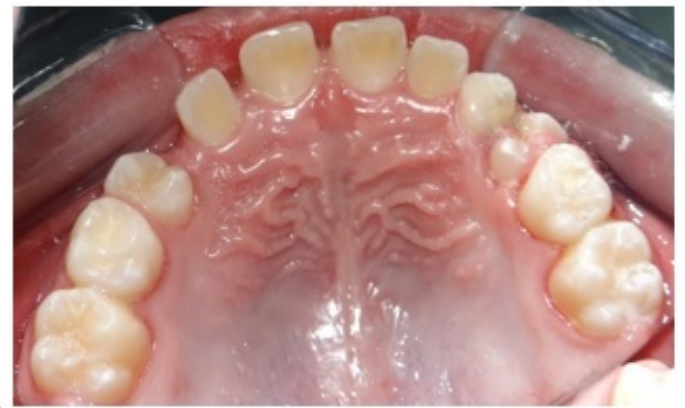

B.

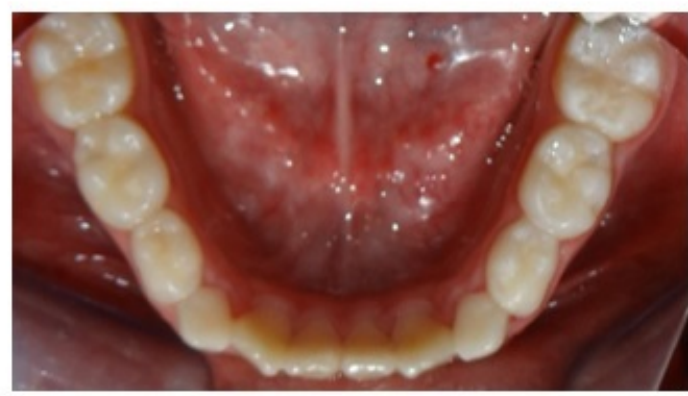

Fig. 6. A) Imagen clínica de arcada superior tras realizar los procedimientos restauradores. B) Imagen clínica de la arcada inferior una vez culminados los procedimientos restauradores.

indicada antes del tratamiento dental. El procedimiento realizado fue el siguiente:

1) Se solicitaron exámenes de laboratorio: biometría hemática y química sanguínea.

2) Se indicó protocolo de profilaxis antibiótica $60 \mathrm{~min}$ antes del procedimiento: Amoxicilina por vía oral a una dosis de $50 \mathrm{mg}$ por cada $\mathrm{Kg} /$ peso.

3) Al inicio de la cita se tomaron los signos vitales

4) Se utilizó anestesia local: 1 cartucho de lidocaína al $2 \%(36 \mathrm{mg})$, con epinefrina a una concentración de 1 : 100,000 .

5) Se realizó la exodoncia del resto radicular del 54 y se colocó una esponja hemostática reabsorbible en el alveólo (Gelatamp), con la finalidad de mantener estabilidad en el proceso de coagulación y adicionalmente obtener un efecto antibacteriano mediante la plata coloidal que contiene el producto. (Fig. 8).

\subsection{Fase de Mantenimiento}

Citas periódicas cada 3 meses con aplicación tópica de agentes fluorados de uso profesional. En cada cita se verificó el nivel del flujo salival en reposo y estimulado, además se realizó la medición de Estreptococo mutans, con pruebas de cultivo. 


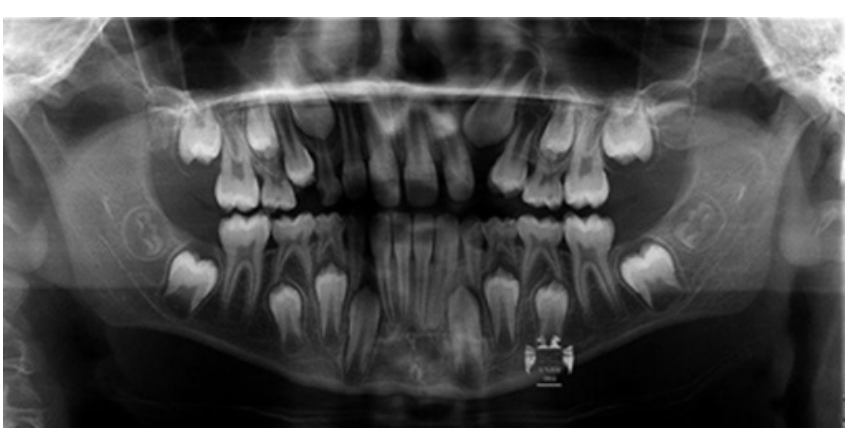

Fig. 7. Ortopantomografía donde se aprecia la presencia de todos los dientes permanentes que están por brotar con buen desarrollo radicular, existe falta de espacio para el brote del 23,33 y 43 .
A.

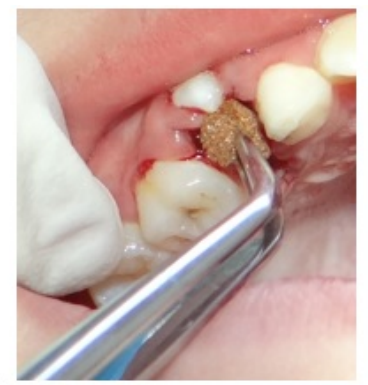

B.

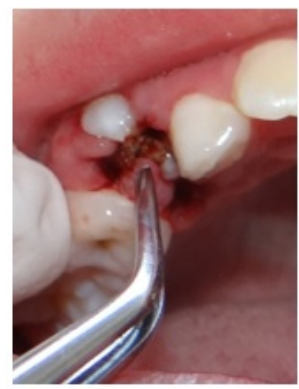

Fig. 8. A) Aplicación inicial de esponja reabsorbible en el alveolo. B) Introducción progresiva y definitiva del Gelatamp en el alveolo

\section{DISCUSIÓN}

En el caso reportado la DMD, fue manejada en la fase médica no ambulatoria temprana, bajo condiciones avanzadas de la enfermedad, con un paciente incapaz de movilizarse e higienizarse por sí mismo. De acuerdo con Symons, ${ }^{15,18}$ el tratamiento dental y las medidas preventivas en los pacientes con DMD deben ser implementados lo más temprano posible, por ello la prioridad número uno fue establecer un protocolo de prevención individualizado teniendo en cuenta las capacidades físicas del niño y sus limitaciones.

Mielnik y Malgorzata, ${ }^{14}$ odontopediátras de la Universidad de Lublin, sugirieron un programa de cuidado de salud oral en el año 2007 para los pacientes con DMD en vista que no existía un protocolo estandarizado para la prevención de caries y enfermedad periodontal en este trastorno. El tratamiento dental preventivo en el Departamento de Odontopediatría UNAM, tomó como base las recomendaciones ya sugeridas por estas autoras y además implementó nuevas estrategias terapéuticas importantes a destacar, se sugirieron y realizaron modificaciones ergonómicas especiales al cepillo dental del paciente, adaptándolo a su condición física y capacidad de manipulación, se complementó la higiene oral con el uso de la seda dental dentro del programa preventivo y adicionalmente se manejó la caries categorizándola bajo normas internacionales de riesgo cariogénico (CAMBRA); combinando la terapia de fluoruro con la quimioterapia de uso casero para mejorar la eficacia del control preventivo.

Desde el punto de vista restaurador, se emplearon técnicas de desproteinización del esmalte antes de colocar los selladores de fosetas, esto con la finalidad de incrementar valores de adhesión del material y asegurar la calidad del tratamiento. Se acoplaron las propiedades del ionómero de vidrio y de las resinas para restaurar los procesos cariosos activos, aprovechando las ventajas independientes de cada material restaurador. Reich y Neff, ${ }^{9}$ sugieren que el manejo dental del paciente con DMD, puede llegar a ser muy complicado debido a problemas de comportamiento, maloclusión e infecciones respiratorias repetidas, dilucidaron claramente en su estudio que el empleo de anestesia general en estos niños está estrictamente contraindicado por el peligro al cual se somete al paciente de desarrollar hipertermia maligna. Basado en este fundamento se realizó el tratamiento ambulatorio en la clínica, con citas cortas y procedimientos rápidos que no fatigaran al niño. El empleo de anestésicos locales con vasoconstrictor adrenérgico estuvo previamente autorizado por el cardiólogo, y se siguió el protocolo estandarizado de profilaxis antibiótica, para proteger al infante en procedimientos que implicaran sangrado. ${ }^{19,20}$ Se extremaron medidas de cuidado en la manipulación de los tejidos, el uso de abrebocas y el empleo de sistemas de aislamiento absoluto. Fue favorable la presencia de la madre durante los tratamientos para incrementar el nivel de aceptación y adicionalmente concienciar a ambos en la importancia de visitar al odontopediatra en forma periódica.

\section{CONCLUSIONES}

La DMD es una condición que cambia con el tiempo, es preciso identificar el tipo de intervenciones terapéuticas que deberán realizarse en cada momento en particular, por ello el odontopediatra, el cual forma parte de un equipo multidisciplinario, debe trabajar activamente en programas y protocolos que garanticen la atención odontológica eficaz y segura. Estos pacientes son vulnerables durante el tratamiento, porque pueden presentar afectación de la capacidad respiratoria, dificultades en la habilidad de comunicación u otro tipo de deficiencias. Para abordarlos se debe disponer de todos los recursos al alcance que permitan una rehabilitación completa y de calidad. Es preciso realizar interconsulta con el cardiólogo en caso que existan alteraciones cardiacas asociadas, evaluando la necesidad de realizar profilaxis antibiótica si el caso lo amerita. Se logró gran éxito clínico, manteniendo la cooperación, continuidad y control en los procedimientos realizados.

\section{Referencias}

1 Ruiz DA, Baumgartner M. Distrofia Muscular de Duchenne (Revisión Bibliográfica), Revista Médica de Costa Rica y Centroamérica. 2008; 586:315-318. 
2 Silva CT, Fonseca DJ, Mateus H, Contreras N, Restrepo CM. Distrofia muscular de Duchenne y Becker Una visión molecular, 2005; 30: 112-116.

3 Chaustre R, Willington CS. Distrofia muscular de Duchenne. Perspectivas desde la rehabilitación, Med. 2011; 19 (1): 45-55.

4 Straub V, Campbell KP. Muscular dystrophies and 20 Tsao CY, Mendell JR: Coexisting muscular dystrophies and the dystrophin-glycoprotein complex, Science Publishers. 1997; 10:168-175.

5 Cammarata-Scalisi F, Camacho N, Alvarado J, Lacruz MA. Distrofia Muscular de Duchenne, presentación clínica, Revista Chilena Pediatrica.2008; 79 (5): 495-501.

6 Ciafaloni E, Fox DJ, Pandya S. Delayed diagnosis in Duchenne Muscular Dystrophy: data from the Muscular Dystrophy Surveillance, Tracking, and Research Network, J Pediatr. 2009; 155(3):380-5.

7 Erazo-Torricelli R. Actualización en distrofias musculares, Revista de Neurología. 2004; 39 (9): 860-871.

8 Morel C, Verdebout, Botteron S, Kiliaridis S. Dentofacial characteristics of growing patients with Duchenne muscular dystrophy: a morphological study, Europ J Orthodont. 2007; 29: 500-507.

9 Reich DR, Neff J. Oral-Surgical management of an odontogenic keratocyst in a patient with Duchenne muscular dystrophy, Pediatric Dentistry.1981; 3 (4):343-345. pacientes con neutropenia febril; revista colombiana de cancerología 2003; 7(4):5-11; citado 20/10/2015

10 Ertürk N, Dogan S. The effect of neuromuscular diseases on the development of dental and occlusal characteristics, Oral Medicine. 1991; 22: 317-321.

11 Bushby K, Finkel R, Birnkrant DJ, et al. Diagnosis and management of Duchenne muscular dystrophy, part 1: diagnosis, and pharmacological and psychosocial management, Lancet Neurol.2010; 9(1):77-93.

12 Kaspar RW, Allen HD, Ray WC, Alvarez CE, Kissel JT, Pestronk A, et al. Analysis of dystrophin deletion mutations predicts age of cardiomyopathy onset in becker muscular dystrophy. Circ Cardiovasc Genet. 2009; 2:544-551.

13 Bushby K, Bourke J, Bullock R, Eagle M, Gibson M, and Quinby J. The multidisciplinary management of Duchenne muscular dystrophy, Current Paediatrics. 2005; 15: 292-300.

14 Mielnik M, Malgorzata B. Duchenne Muscular Dystrophy a dental healthcare program, Spec Care Dentist.2007; (27): 23-25.

15 Symons AL, Townsend GC, Hughes TE. Dental Characteristics of patients with Duchenne muscular dystrophy, ASDC J Dent Child.2002; 69:277-83.

16 Fonseca D, Tamar C, Mateus H: Detección de portadoras de distrofia muscular de Duchenne en familias colombianas mediante análisis de microsatélites. Colomb Med 2008; 39: 7-13.

17 Erazo Torricelli R. Actualización en distrofias musculares. Rev Neurol 2004; 39: 860-71.

18 Longo Araújo de Melo E, Moreno Valdés MT. Buena ca- lidad de vida de los pacientes con distrofia muscular de Duchenne. Rev Neurol 2007; 45: 81-7.

19 American academy of pediatrics section on cardiology and cardiac surgery. Cardiovascular health supervision for individuals affected by Duchenne or Becker muscular dystrophy. Pediatrics 2005; 116:1569-73. epilepsy in children. J Child Neurol 2006; 21:148-50.

Recibido: 05 de Enero de 2016

Aceptado: 17 de Enero de 2016 